OPEN ACCESS

Edited by:

Rongming Liu,

University of Colorado Boulder,

United States

Reviewed by:

Chong Zhang,

Tsinghua University, China

Feng $L i$,

Tianjin University, China

${ }^{*}$ Correspondence:

Kequan Chen

kqchen@njtech.edu.cn

Specialty section:

This article was submitted to

Synthetic Biology,

a section of the journal

Frontiers in Bioengineering and

Biotechnology

Received: 02 August 2020 Accepted: 24 September 2020

Published: 15 October 2020

Citation:

Feng J, LU Q, Li K, Xu S, Wang X,

Chen K and Ouyang P (2020)

Construction of an Electron Transfer

Mediator Pathway

for Bioelectrosynthesis by Escherichia

\section{Construction of an Electron Transfer Mediator Pathway for Bioelectrosynthesis by Escherichia coli}

\author{
Jiao Feng, Qiuhao Lu, Kang Li, Sheng Xu, Xin Wang, Kequan Chen* and Pingkai Ouyang \\ State Key Laboratory of Materials-Oriented Chemical Engineering, College of Biotechnology and Pharmaceutical \\ Engineering, Nanjing Tech University, Nanjing, China
}

Microbial electrosynthesis (MES) or electro-fermentation (EF) is a promising microbial electrochemical technology for the synthesis of valuable chemicals or high-value fuels with aid of microbial cells as catalysts. By introducing electrical energy (current), fermentation environments can be altered or controlled in which the microbial cells are affected. The key role for electrical energy is to supply electrons to microbial metabolism. To realize electricity utility, a process termed inward extracellular electron transfer (EET) is necessary, and its efficiency is crucial to bioelectrochemical systems. The use of electron mediators was one of the main ways to realize electron transfer and improve EET efficiency. To break through some limitation of exogenous electron mediators, we introduced the phenazine-1-carboxylic acid (PCA) pathway from Pseudomonas aeruginosa PAO1 into Escherichia coli. The engineered E. coli facilitated reduction of fumarate by using PCA as endogenous electron mediator driven by electricity. Furthermore, the heterologously expressed PCA pathway in E. coli led to better EET efficiency and a strong metabolic shift to greater production of reduced metabolites, but lower biomass in the system. Then, we found that synthesis of adenosine triphosphate (ATP), as the "energy currency" in metabolism, was also affected. The reduction of menaquinon was demonstrated as one of the key reactions in self-excreted PCAmediated succinate electrosynthesis. This study demonstrates the feasibility of electron transfer between the electrode and E. coli cells using heterologous self-excreted PCA as an electron transfer mediator in a bioelectrochemical system and lays a foundation for subsequent optimization.

Keywords: electron transfer mediator, phenazine-1-carboxylic acid, Escherichia coli, bioelectrocatalysis, succinate electrosynthesis

\section{INTRODUCTION}

Microbial electrosynthesis (MES) or electro-fermentation (EF) relies on microbial cells as catalysts to increase terminal production of high value fuels and chemicals via electricity (Schroder et al., 2015). They provide a promising strategy for the conversion of electrical energy (current) to chemical energy (extracellular multi-carbon products) to achieve energy storage and distribution and therefore have been widespread concerned. Initially, ancetate was produced from the reduction of $\mathrm{CO}_{2}$ with the help of MES (Nevin et al., 2010). Among the developing microbial electrochemical 
technologies currently known, MES and EF have widespread applications in optimization of more high-value terminal metabolite production from $\mathrm{CO}_{2}$ or other substrates (Wang and Ren, 2013; Ganigue et al., 2015; Wu et al., 2019). In bioelectrochemical reactors, microbial cells grow and catalyze reactions at high overpotentials (negative cathode potential) during MES or EF operation. Driven by an electrical current, microbial cells interact with an electrode through a series of oxidation/reduction reactions and electron transfer reactions to stimulate and change the substantial metabolism, energy metabolism or microbial growth (Rosenbaum et al., 2011). It is also able to generate intracellular reducing equivalents by using the electrons, which offers a possible route to break through the limitation of metabolic redox state/balance and energy carriers (Harrington et al., 2015a). Therefore, the primary task is to create cell-electrode connections with inward EET pathway and crucial to MES is the ability and efficiency of microbes to perform the EET process of electron transfer from extracellular redox-active electron donors to intracellular acceptors.

An often-used and attractive method to realize electron flow from extracellular electrodes into microorganisms is the use of electron mediators in bioelectrochemical systems. The electron mediators could be used by both suspended microorganisms in the medium and immobilized microorganisms at the electrode (Patil et al., 2012). Among the electron mediators employed, artificial exogenous mediators like neutral red (Harrington et al., 2015a), methyl viologen (Aulenta et al., 2007), and 2,4-anthraquinonedisulfate (Thrash et al., 2007) have been successfully used in bioelectrochemical systems to yield more valuable fuels and reduced chemical or treat wastewater, and perform well in electron transfer. However, artificial exogenous mediators also have some disadvantages, such as exhibiting limited stability, having short lifespans, being costly to implement, and their uncertainty of sustainability in largescale applications (Patil et al., 2012). Thus, developing costefficient and sustainable electron mediator that does not require continuous addition is one of major challenges. In contrast to this method of exogenous addition of artificial electron mediators, self-secreted electron mediators derived from microorganisms could overcome this challenge.

The currently reported self-secreted electron mediators are mostly produced and secreted by electrochemically active bacteria $(\mathrm{EAB})$ to facilitate electron transfer from the cathode. Examples include pyrroloquinoline quinone secreted by Acinetobacter calcoaceticus NBRC12552 (Freguia et al., 2010) and self-secreted flavins by Shewanella loihica PV-4 (Liu et al., 2012). Although direct electron transfer from cathodes to microorganisms has been attributed to the Mtr pathway in Shewanella oneidensis MR-1, increased production of electron mediators (flavins) have been shown to promote an inward current (Yang et al., 2015). It also has been reported that mediated electron transfer is most important mode of EET. While $\mathrm{EAB}$ has excellent ability of self-secreted electron mediators, further application on EAB is limited by the narrow spectrum of substrates and simple compounds that can be synthesizes. And their sophisticated molecular biology tools and available synthetic biological toolsets are not mature. Conversely, model microbe like Escherichia coli possess many advantages in genetic modifications and chemical biosynthesis.

Here, we heterologously introduced the synthesis pathway of electron mediators into the model microbe, E. coli, to exploit the advantages of electron mediators while simultaneously alleviating the limitations of EAB. Phenazine-1-carboxylic acid (PCA) is a type of redox-active phenazine and is produced by species of Pseudomonas and Actinomycetes. PCA possesses a relatively low for redox potential $(E=-0.24 \mathrm{~V}$ vs. $\mathrm{Ag} / \mathrm{AgClsat})$ and is primarily involved in reversible redox cycling under oxygen-limited conditions (Bosire et al., 2016; Bosire and Rosenbaum, 2017). Reportedly, PCA rather than pyocyanin may be responsible for electron transfer from the cathode to Pseudomonas aeruginosa PA14 (Bosire and Rosenbaum, 2017). In our previous our work, we established that PCA could perform the role of electron transfer and effectively improve current generation in microbial fuel cell by introducing the PCA pathway of $P$. aeruginosa in E. coli (Feng et al., 2018). PCA production remained relatively stable and the concentration of self-excreted PCA increased with the operation of bioelectrochemical reactor (Feng et al., 2018). However, critical metabolic differences between E. coli and $P$. aeruginosa make it ambiguous whether self-secreted heterologous PCA would be utilized in MES performed by E. coli and unknown are the effects it might have on growth and metabolism under the electrochemical tension.

In this study, we investigated how the introduction of the PCA pathway of $P$. aeruginosa into $E$. coli affects PCA production and viability. Furthermore, the heterologously expressed PCA pathway was employed to transfer electrons from the cathode to the microbial cells and achieve reduction of fumarate in bioelectrochemical reactors at negative cathode potential. Through the measurement of electrochemical parameters and fermentation products, the effect of the heterologously expressed PCA pathway on metabolism of E. coli and bioelectrocatalytic activity under the electrochemical tension was analyzed. Additionally, we found that adenosine triphosphate (ATP), as the "energy currency," was stimulated by the electrical current through the cathode provided by the inward EET process utilizing the heterologously self-excreted PCA in E. coli.

\section{MATERIALS AND METHODS}

\section{Bacterial Strains, Plasmids and Plasmid Construction}

The strains BA102 and PAO1 of E. coli and P. aeruginosa, respectively, were used in this work (Table 1). Plasmids and construction of plasmids are described in our previous study (Feng et al., 2018, 2020). Recombinant plasmid ptrc99a-phzA1G1 was transformed into E. coli BA102 to obtain a PCAproducing strain (designated as E. coli-phz). The deletion strain E. coli BA102 ( $\triangle m e n A)$ was constructed as described previously using the method of the CRISPR-Cas9 System (Feng et al., 2020). Firstly, the competent cells of E. coli BA102 harboring pCas were prepared as described previously by Jiang et al. (2015). Then donor DNA of menA and plasmid pTargetF-C-menA were constructed through PCR using the corresponding primers listed 
TABLE 1 | Strains, plasmids, and primers.

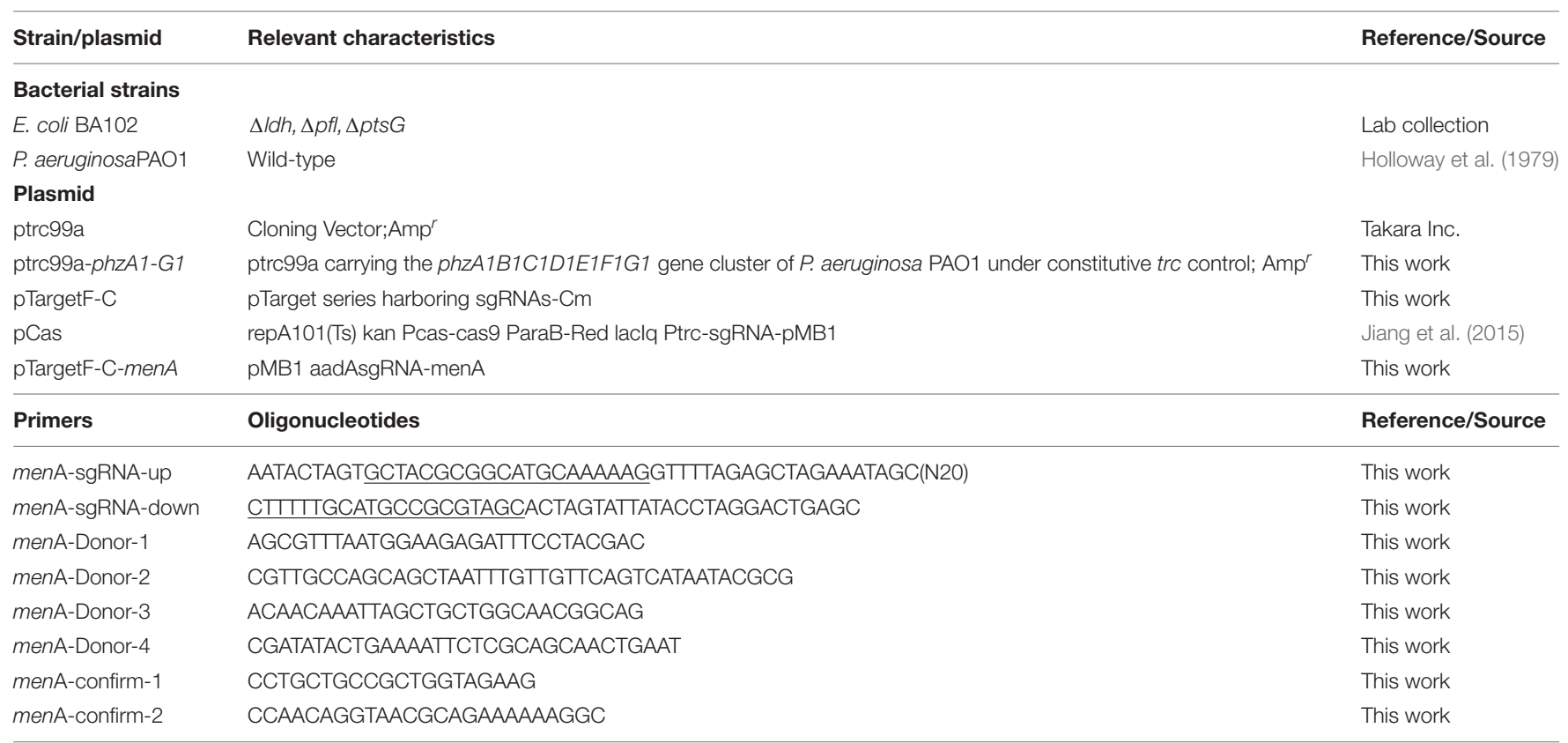

sgRNA-menA: sgRNA with an N20 sequence for targeting the menA locus.

in Table $\mathbf{1}$ and E. coli BA102 genome and pTargetF-C as the template, respectively. The pTargetT-menA (100 ng) and donor DNA (500 ng) were mixed and then added to a total of $50 \mu \mathrm{l}$ of competent cells. Introduction of the pTargetT-menA and DNA into $E$. coli cells was carried out by electroporation at $2.5 \mathrm{kV}$. After being inoculated in $1 \mathrm{~mL}$ Luria-Bertani (LB) medium at $30^{\circ} \mathrm{C}$ for $1 \mathrm{~h}$, cells were harvested by centrifugation $(4000 \mathrm{rpm})$ for $3 \mathrm{~min}$, spread onto LB agar plates and then incubated overnight at $30^{\circ} \mathrm{C}$. $50 \mu \mathrm{g} / \mathrm{ml}$ kanamycin and $34 \mu \mathrm{g} / \mathrm{mL}$ chloramphenicol were added in LB agar plates for selection. Finally, transformants were identified by PCR using menA-confirm-1/menA-confirm-2 as primers and gene sequencing. The plasmid ptrc99a-phzA1-G1 was transformed into E. coli BA102 ( $\Delta m e n A)$ to obtain E. coli-phz ( $\triangle m e n A)$.

\section{Media and Growth Conditions}

Samples of E. coli and $P$. aeruginosa stored at $-80^{\circ} \mathrm{C}$ were pregrown for $8-12 \mathrm{~h}$ at $37^{\circ} \mathrm{C}$ in $\mathrm{LB}$ medium. Then each culture suspension $(1 \%)$ was used to inoculate $100 \mathrm{~mL} \mathrm{LB}$ medium and cultured at $37^{\circ} \mathrm{C}$ and $200 \mathrm{rpm}$. When E. coli-phz grew to $\mathrm{OD}_{600}$ about 0.6, an inducer (IPTG) was added for phzA1-G1 expression. After an additional 10-12 h of growing, cultures were saved for further analysis and electrochemical measurements. For E. coliphz, ampicillin $(100 \mu \mathrm{g} / \mathrm{mL})$ was added into its LB medium in order to maintain selection for cells with the plasmid ptrc99aphzA1-G1 in E. coli.

\section{Construction and Operation of Bioelectrochemical Reactor}

A dual chamber bioelectrochemical reactor (internal volume per chamber of $250 \mathrm{ml}$ ) was constructed. The two reaction chambers were separated by a proton exchange membrane
(Nafion, diameter, $35 \mathrm{~mm}$, DuPont Co., United States). The cathode and anode electrodes were made of fine carbon felts ( $5 \mathrm{~mm}$ thick; $80 \mathrm{~mm} \times 40 \mathrm{~mm}$ ). The cathode was imposed at $-0.6 \mathrm{~V}$ using an $\mathrm{Ag} / \mathrm{AgCl}$ electrode as the reference electrode. The anode medium was made up of the following: $35.8 \mathrm{~g} \cdot \mathrm{L}^{-1} \mathrm{NaHPO}_{4} \cdot 12 \mathrm{H}_{2} \mathrm{O}, 15.6 \mathrm{~g} \cdot \mathrm{L}^{-1} \mathrm{NaH}_{2} \mathrm{PO}_{4} \cdot 2 \mathrm{H}_{2} \mathrm{O}$, and $5.8 \mathrm{~g} \cdot \mathrm{L}^{-1} \mathrm{NaCl}$, adjust to $\mathrm{pH} 7.2$ with $\mathrm{NaOH}$. We filtered dithiothreitol (DTT, $0.02 \%$ ) through a $0.22 \mathrm{~nm}$ syringe filter and then added it into the anode medium before use. The cathode medium contained: $31.5 \mathrm{~g} \cdot \mathrm{L}^{-1} \mathrm{NaHPO}_{4} .12 \mathrm{H}_{2} \mathrm{O}, 8.5 \mathrm{~g} \cdot \mathrm{L}^{-1}$ $\mathrm{NaH}_{2} \mathrm{PO}_{4} \cdot 2 \mathrm{H}_{2} \mathrm{O}, 10.0 \mathrm{~g} \cdot \mathrm{L}^{-1} \mathrm{NaHCO}_{3}$, and $5.0 \mathrm{~g} \cdot \mathrm{L}^{-1}$ yeast extract. Prior to inoculation, sodium fumarate or D-glucose was added into the cathode medium and the $\mathrm{pH}$ of the cathode medium was adjusted to approximately 7.0 through flushing with $\mathrm{CO}_{2}$. The bacterial culture and cathode medium were mixed in a ratio of 1:2 and then inoculated into the cathode chamber. $\mathrm{NaHCO}_{3}$ and gaseous $\mathrm{CO}_{2}$ were added into bioelectrochemical reactors as carbon sources for MES. Bioelectrochemical reactors and serum bottles were stirred using a magnetic stirrer at $200 \mathrm{rpm}$ and kept in incubators at $35 \pm 1^{\circ} \mathrm{C}$. The headspace of the cathode chamber inserted an airtight syringe to take samples regularly.

\section{Cell Growth and Quantification of ATP}

The cell density was tracked via absorbance $\left(\mathrm{OD}_{600 \mathrm{~nm}}\right)$ by using a UV-visible spectroscopy system. The injection volume was $20 \mu \mathrm{l}$. A BacTiter-Glo ${ }^{\mathrm{TM}}$ Microbial Cell Viability Assay (Promega, G8230, United States) was used to determine the concentrations of ATP.

\section{Organic Acid and PCA Analysis}

The organic acid concentrations were assayed by an HPX$87 \mathrm{H}$ column $(300 \mathrm{~mm},-7.8 \mathrm{~mm}$, Bio-Rad, Hercules, CA, United States) using a high-performance liquid chromatography 
(HPLC) system (Agilent Technologies, Santa Clara, CA, United States). HPLC was equipped with a refractive index detector set at $35^{\circ} \mathrm{C}$ and an ultraviolet spectrophotometric detector monitored at $215 \mathrm{~nm}$. The column temperature was set to $60^{\circ} \mathrm{C}$. The mobile phase was $8 \mathrm{mM}$ sulfuric acid at a flow rate of $0.5 \mathrm{~mL} / \mathrm{min}$.

Phenazine-1-carboxylic acid was extracted from the culture supernatants according to a previously reported method (Feng et al., 2018) and analyzed by using HPLC system, which was equipped with an ultraviolet spectrophotometric detector set at $280 \mathrm{~nm}$ and a C18 analytical column (5 mm particle size, $250 \mathrm{~mm}$, $4.6 \mathrm{~mm}$ i.d.) (Yong et al., 2011; Feng et al., 2018). The mobile phases were water with $0.05 \%$ acetic acid and acetonitrile with $0.05 \%$ acetic acid, respectively. The chromatographic separations utilized a linear gradient from $15 \%$ acetonitrile with $0.05 \%$ acetic acid to $83 \%$ within $20 \mathrm{~min}$, followed by $15 \%$ acetonitrile with $0.05 \%$ acetic acid from 20 to $25 \mathrm{~min}$ at a flow rate of $1 \mathrm{~mL} / \mathrm{min}$.

\section{Electrochemical Analysis}

Cyclic voltammogram (CV) measurements were performed by an electrochemical instrument (PMC 1000/DC, AMETEK, United States) in a three-electrode configuration using an $\mathrm{Ag} / \mathrm{AgCl}$ electrode as the reference electrode. $\mathrm{CV}$ measurements were employed with a scan range of -800 to $600 \mathrm{mV}$ and a scan rate of $20 \mathrm{mV} / \mathrm{s}$.

Electrochemical impedance spectroscopy (EIS) was measured to the Nyquist plots of impedance on PMC 1000/DC and the frequency was decreased at an amplitude of $10 \mathrm{mV}$ from $10^{5}$ to 0.01 .

\section{RESULTS AND DISCUSSION}

\section{Successful Introduction of the Heterologous PCA Pathway Into E. coli}

The engineered E. coli-phz was constructed by transforming ptrc99a-phzA1-G1 into E. coli BA102. The parental strain E. coli BA102 was constructed by disabling the $p f B$ gene, $l d h A$ gene, and $p t s G$ gene to eliminate main competing fermentation pathways and improve succinate production. The culture medium of E. coli-phz after $12 \mathrm{~h}$ of culturing appeared yellow (Supplementary Figure 1), resulting from the color of the pigment of the phenazine PCA. Similar to the data presented by our previous reports (Feng et al., 2018), a significant peak of PCA was found in the extracts of E. coli-phz, while no PCA synthesis was observed in E. coli BA102 (Supplementary Figure 2). These results reflect that the phzA1-G1 gene from $P$. aeruginosa PAO1 was efficiently expressed and PCA was synthesized in E. coliphz.

To investigate the effect of heterologous PCA on cell growth, PCA production, and $\mathrm{OD}_{600 \mathrm{~nm}}$ values were assessed by titration of various IPTG concentrations ranging from 0 to $0.75 \mathrm{mM}$. With increasing IPTG concentration, PCA production also gradually increased which demonstrated that PCA was synthesized to $3.87 \mathrm{mg} / \mathrm{L}$ in E. coli even relying on basal expression (Jensen et al., 2010). However, cell growth was gradually inhibited as shown in Figure 1. Of the E. coli BA102 and E. coli-phz strains separately

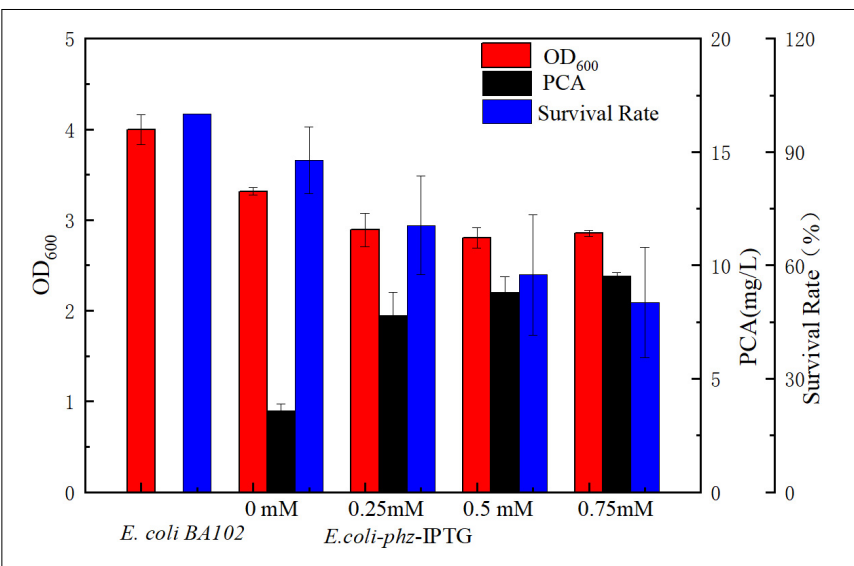

FIGURE 1 | The effect of phzA1-G1 gene expression on PCA production (black) and cell growth (red) at the aerobic growth stage and survival rate (blue) of cells in the bioelectrochemical system at $8 \mathrm{~h}$. Cell survival rate shows the changes of $\mathrm{OD}_{600 \mathrm{~nm}}$ at $8 \mathrm{~h} / \mathrm{OD}_{600 \mathrm{~nm}}$ at $\mathrm{O} \mathrm{h}$ in the bioelectrochemical system compared with that of $E$. coliBA102.

inoculated into the bioelectrochemical reactor and imposed at $-0.6 \mathrm{~V}$ (vs. Ag/AgCl), both presented decreases of cell number in the first $8 \mathrm{~h}$. The survival rate of E. coli-phz decreased from 87.86 to $57.51 \%$ as the IPTG concentrations increased from 0 to $0.5 \mathrm{mM}$ in the bioelectrochemical system (Figure 1). The results indicate that the electrical current might have led to cellular damage. To ensure the presence of a sufficient amount of living cells, all subsequent experiments were performed in the absence of IPTG.

\section{The Function of Self-Excreted PCA as the Endogenous Electron Mediator in the Bioelectrochemical System Driven by Electricity}

To test the function of the endogenous electron shuttle PCA in an bioelectrochemical system, the reaction of fumarate reduction to succinate was carried out, which is a simple onestep reaction with electrons transfer and an essential catalyzed reaction for microbes (Fang et al., 2020). The self-excreted PCA did not show significantly high efficiency. Some exogenous electron mediators, such as neutral red, 2,6-dichloroindophenol, potassium ferricyanide, and Lauth's violet also showed low efficiency in MES by E. coli according to previous report (Wu et al., 2019). In the bioelectrochemical systems, cellular damage caused by the electrical current led to a reduced survival rate of E. coli-phz (Figure 1). Considering the difference in cell density between E. coli-phz and E. coli BA102, the catalytic effect of per unit E. coli-phz cells (succinate concentration/ $\mathrm{OD}_{600}$ ) was investigated. As the results illustrate in Table 2, the succinate/ $\mathrm{OD}_{600}$ of $E$. coli-phz was up to $137 \%$, compared with that of $E$. coli BA102 cells. The ability of PCA to transfer electrons likely promoted the succinate production of per unit E. coli$p h z$ cells. We conclude that self-excreted PCA could transfer electrons between the cell and the electrode by introducing the heterologous PCA pathway into E. coli. 
TABLE 2 | Fermentation profiles in bioelectrochemical systems of E. coli BA102 and E. coli-phz.

\begin{tabular}{|c|c|c|c|c|c|c|}
\hline Strains & Succinate $^{\star}(g / L)$ & Succinate $(g / L)$ & Acetate (g/L) & Pyruvate (g/L) & Initial $O_{600 \mathrm{~nm}}$ & Terminated $O_{600 \mathrm{~nm}}$ \\
\hline E. coli BA102 & $3.85 \pm 0.20$ & $6.93 \pm 0.26$ & $1.57 \pm 0.52$ & $2.88 \pm 0.33$ & $1.05 \pm 0.10$ & $1.53 \pm 0.31$ \\
\hline E. coli-phz & $5.26 \pm 0.38$ & $7.26 \pm 0.22$ & $1.21 \pm 0.23$ & $1.98 \pm 0.28$ & $0.90 \pm 0.02$ & $1.01 \pm 0.13$ \\
\hline
\end{tabular}

Succinate*: succinate concentration/OD 600 with fumarate as the substrate.

\section{Heterologous PCA Pathway Enhanced Bioelectrocatalytic Activity}

In order to elucidate the effect of the heterologous PCA pathway on bioelectrocatalytic activity, CV and EIS measurements were obtained. $\mathrm{CV}$ was used to investigate the redox reactions at the microbe-electrode interface to reveal the characterization of the bioelectrocatalysis (Liu et al., 2005). The redox species that took part in EET in the electrochemical systems were determined by $\mathrm{CV}$ analysis. The $\mathrm{CV}$ data indicated that there was no significant redox peak in the bioelectrochemical system inoculated with E. coli BA102 (Figure 2A), which showed that there was no significant redox reactions between E. coli BA102 cells and electrode. In contrast to E. coli BA102, a significant pair of redox peak was observed from the bioelectrochemical system inoculated with E. coli-phz. Figure 2A showed an oxidation peak in the range of 0.0 to $-0.20 \mathrm{~V}$ and reduction peak between -0.30 and $-0.50 \mathrm{~V}$, which agreed with the broad PCA redox peak system determined for P. aeruginosa PA14 (Bosire et al., 2016). The results indicate that self-excreted PCA functioned as electron mediator in the bioelectrochemical system inoculated with E. coli-phz, which achieved the utilization of electrons and redox reaction in the bioelectrochemical system inoculated with E. coli-phz.

Nyquist plots of impedance obtained by EIS measurements can precisely evaluate the electron transfer resistance of $E$. coliphz and E. coli BA102 (He and Mansfeld, 2009; Nandy et al., 2016). The diameter of the well-defined semicircle curve in the high frequency region was equivalent to the electron transfer resistance. As shown in Figure 2B, the charge transfer resistance of the E. coli-phz system was lower than that of E. coli BA102 system, indicating there was a higher electron transfer rate in E. coli-phz. These results demonstrate that self-excreted PCA in E. coli-phz, accomplished by introducing the heterologous PCA pathway in E. coli, was the main electron mediator employed in electron transfer reactions that improved the EET between microbial cells and the electrode.

\section{Heterologous PCA Pathway Affected the Central Metabolism With Glucose as the Carbon Source in Bioelectrochemical System}

In previous experiments, researchers have successfully increased conversion of succinate from fumarate in MES (Harrington et al., 2015b; Wu et al., 2019; Fang et al., 2020). Microbial electrosynthesis offers a possible route to move beyond the limitation of metabolic redox state and disturb intracellular metabolism. To better understand how the heterologous PCA pathway can alter cell growth and a metabolism driven by electrical current, glucose (final concentration of $10 \mathrm{~g} / \mathrm{L}$ ) was used as the carbon source. Fermentation products, including succinate, pyruvate and acetate were measured by HPLC to investigate changes in cellular metabolism. Succinate was produced at about $7.26 \mathrm{~g} / \mathrm{L}$ by E. coli-phz and $6.93 \mathrm{~g} / \mathrm{L}$ by E. coli BA102 after $45 \mathrm{~h}$ in culture (Table 2). The final optical density $\left(\mathrm{OD}_{600}\right)$ of E. coli-phz was lower than that of E. coli BA102, which suggested that cell growth of E. coli-phz was inhibited compared to the increasing cell growth of E. coli BA102. This demonstrates that the production capacity of succinate per unit of E. coli-phz was higher than that of $E$. coli BA102. The higher titer of the reduced production was in good agreement with the result of MES by E. coli with NR as exogenous electron mediator, which was due to the utilization of electricity (Harrington et al., 2015a; Wu et al., 2019). Since PCA was self-excreted by E. coli-phz in this work, the problems on exogenous addition and the cost were dealt with.

In addition, because of the $p f l B$ deletion, pyruvate accumulated as one of main by by-products. As shown in Table 2, E. coli-phz displayed a greater ratio of succinate/pyruvate and succinate/acetate contents than those of E. coli BA102. The results demonstrate that self-secreted PCA of E. coli-phz could transfer electrons from a cathode to intracellular electron carriers. Furthermore, microbial cells were stimulated and changed the intracellular metabolite profiles to facilitate the production of reduced metabolites driven by electrical current.

\section{The Limiting Factor of $E$. coli Cells in Bioelectrochemical System}

It is well known that microbial cells in bioelectrochemical system (MES and EF) interact with the electrode through a series of oxidation/reduction and electron transfer reactions to drive the biocatalysis reactions or supply electrons from the cathode. In addition, some research have shown that intracellular energy metabolism, biological activity and biofilm formation can be stimulated and changed by providing electrical currents through cathodes (Yong et al., 2011). In our previous experiments, we observed retarded cell-growth in a bioelectrochemical system inoculated with E. coli-phz compared to the inoculated with E. coli BA102. According to previous literature reports, the growth of $P$. aeruginosa was inhibited by electrical stimulation (both cathodal and anodal stimulation) (Kincaid and Lavoie, 1989; Szuminsky et al., 1994; Asadi and Torkaman, 2014). In bioelectrochemical system, the cell growth or biomass generation is related to the molecular mechanisms of ET from the cathode to terminal electron acceptor in the microbes (Harrington et al., 2015b). As Clark and Harrington et al. have indicated, the major reaction in the inner membrane of $E$. coli is menaquinone $(\mathrm{MK})$ reduction in mediated bioelectrochemical 

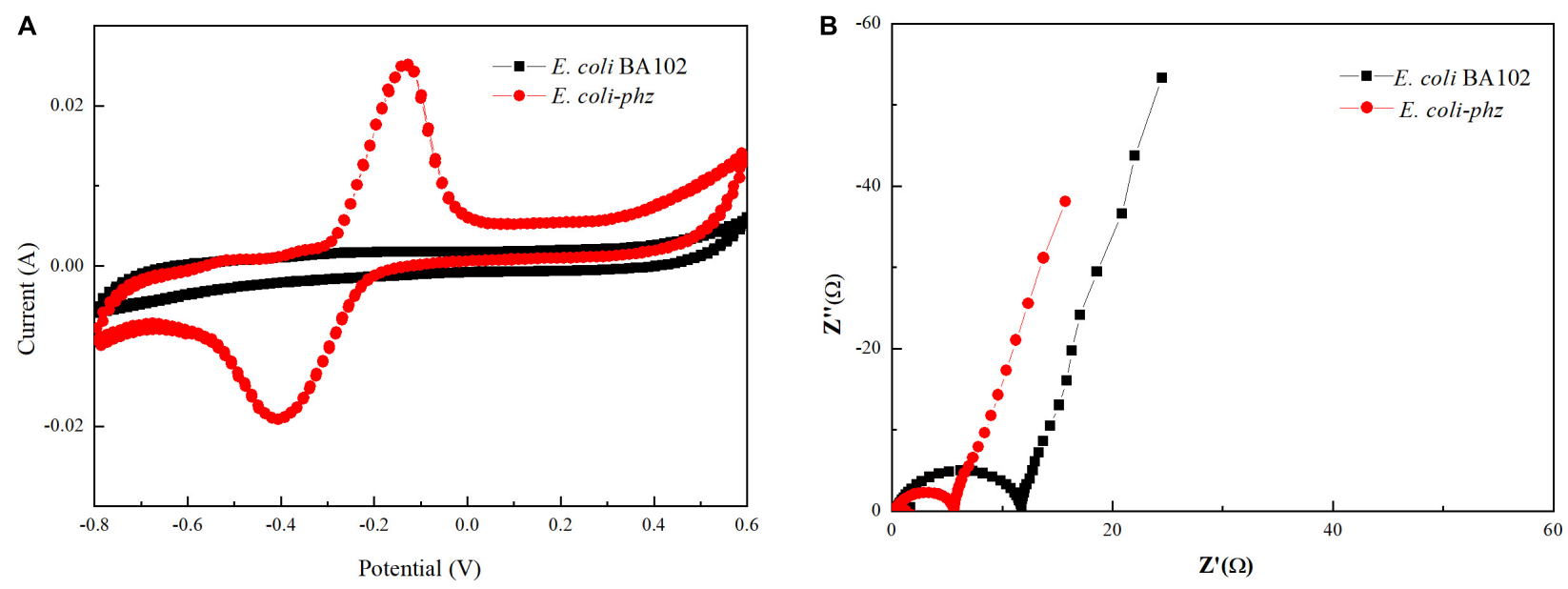

FIGURE 2 | Effects of self-excreted PCA on bioelectrocatalytic activity in bioelectrochemical system. Cyclic voltammetry (A) and Nyquist plots (B). E. coli BA102 (black square) and E. coli-phz (red circle).

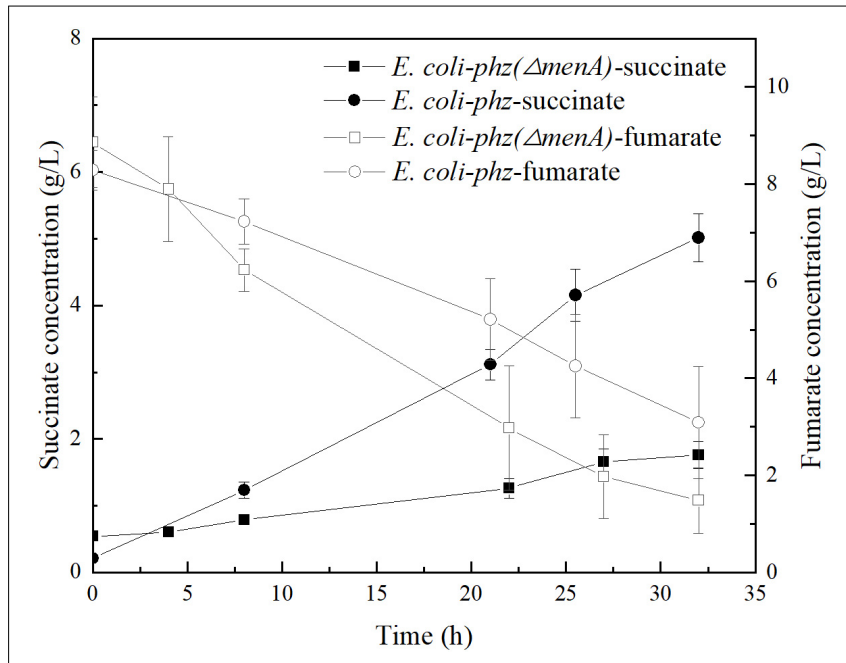

FIGURE $\mathbf{3}$ | The concentrations of succinate and fumarate in the bioelectrochemical systems of E. coli-phz (black circle) and E. coli-phz( $\triangle m e n A)$ (black square). system (Clark et al., 2012; Harrington et al., 2015b). In NRmediated bioelectrochemical system, $\mathrm{NRH}_{2}$ gains electrons from the cathode and diffuses into the inner membrane, where $\mathrm{MK}$ is reduced to $\mathrm{MKH}_{2}$ by $\mathrm{NRH}_{2}$. $\mathrm{MKH}_{2}$ then passes electrons to the terminal reductase with concomitant reduction of the electron acceptor to reduced end-product (Harrington et al., 2015b). In order to test the role that MK play in self-excreted PCA-mediated electrosynthesis, the $m e n A$ deletion strain E. coli BA102 ( $\triangle m e n A$ ) was constructed and ptrc99a-phzA1-G1 was transformed into E. coli BA102 ( $\triangle m e n A)$ to obtain E. coli-phz ( $\triangle m e n A)$. Fumarate was added to the bioelectrochemical system culture of $E$. coli$p h z(\triangle m e n A)$ to compare the consumption of fumarate and the accumulation of succinate with E. coli-phz, respectively. As shown in Figure 3, disabling the $m e n A$ gene resulted in the normal

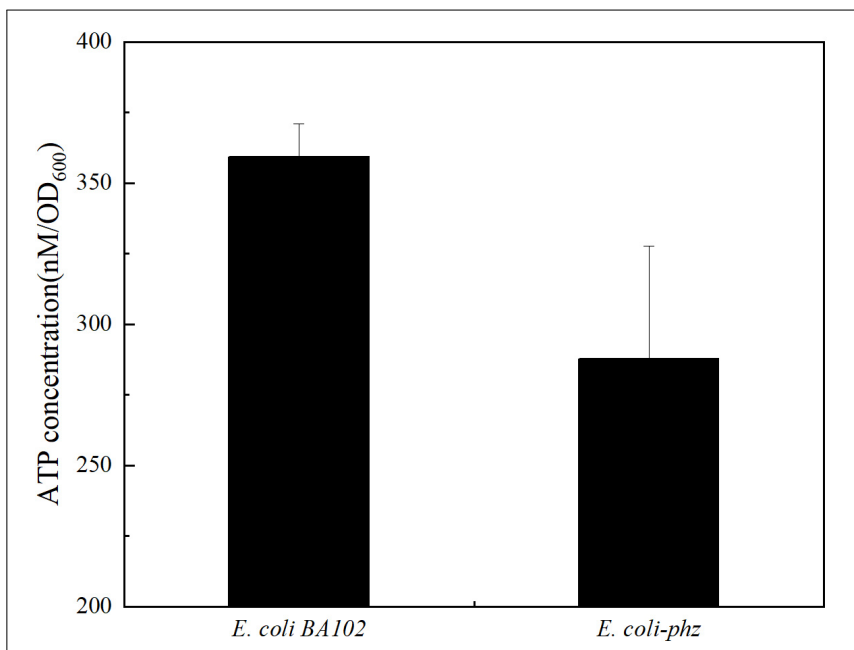

FIGURE 4 | The ATP levels of E. coli BA102 and E. coli-phz.

consumption of fumarate but significantly lower accumulation of succinate in MES system. This suggests that MK was critical to the reduction of fumarate to succinate in bioelectrochemical system of $E$. coli. The first relay station of fumarate reductase to use exchange electrons with quinines has been confirmed to be the center3[3Fe-4S] cluster of fumarate reductase (Heering et al., 1997). Given these results, MK is one of the key components in PCA-mediated electrosynthesis.

According to the hypothesis, electrons of $\mathrm{MKH}_{2}$ are derived from reduced PCA instead of NADH via NADH dehydrogenase (Complex I) partly, resulting in lower ATP yields and growth inhibition. The reaction that $\mathrm{MK}$ is reduced by $\mathrm{NADH}$ via Complex I can contribute the proton motive force, which is used to drive ATP synthase to synthesize ATP. If reductant is PCA, the proton motive force was not generated. ATP is often referred to as the "energy currency" in all living microbial cells that supplies 
energy for cellular functions and processes. It is also useful in research as an independent bio-indicator of microbial metabolic viability and activity. To test the hypothesis, we measured ATP contents and observed a lower level of ATP in E. coli-phz than E. coli BA102 in bioelectrochemical systems (Figure 4). To further verify the effects of ATP on the biomass and the production of succinate, ATP was exogenously added in the bioelectrochemical system inoculated with $E$. coli-phz. The results showed that the terminated $\mathrm{OD}_{600}$ of E. coli-phz increased by $19.4 \%$ by the exogenous addition of ATP, but there was not an increase in succinate production compared with that of the control group without addition of ATP (Supplementary Figure 3). The results suggested that there were other factors affect biomass generation and succinate production, such as instable chemical property of ATP, the low external redoxpotential environment (Wu et al., 2019), intracellular unbalanced redox state (Heux et al., 2006; Liang et al., 2013) induced by the transfer and utilization of the cathode electrons. To achieve more efficient electrosynthesis, screening of the electricity-tolerance strain would be carried out and the electrosynthesis conditions would be further optimized.

\section{CONCLUSION}

Escherichia coli BA102 can generate and excrete PCA after being transformed to express phzA1B1C1D1E1F1G1 from $P$. aeruginosa PAO1. Engineered E. coli in bioelectrochemical system using self-excreted PCA as electron transfer mediator allowed electrons on the cathode to be transferred to the inner membrane, leading to the connection between the electrode and microbial cells and improvement of electron transfer efficiency. Driven by the electrical current, the influence of a heterologous electron transfer mediator on cell growth, fermentation production, and bioelectrocatalytic activity of E. coli cells was studied. In the self-excreted PCA-mediated bioelectrochemical system, the electrical current led to cellular damage and affected cell growth. However, succinate production increased and by-products declined. The results demonstrated that the electrical current of E. coli-phz altered the metabolism to facilitate the production of reduced metabolites. Meanwhile, synthesis of ATP, the "energy currency," was stimulated by the electrical current through the cathode. It also found that $\mathrm{MK}$ was critical to the reduction of fumarate to

\section{REFERENCES}

Asadi, M. R., and Torkaman, G. (2014). Bacterial Inhibition by electrical stimulation. Adv. Wound Care 3, 91-97.doi: 10.1089/wound.2012.0410

Aulenta, F., Catervi, A., Majone, M., Panero, S., Reale, P., and Rossetti, S. (2007). Electron transfer from a solid-state electrode assisted by methyl viologen sustains efficient microbial reductive dechlorination of TCE. Environ. Sci. Technol. 41, 2554-2559.doi: 10.1021/es0624321

Bosire, E. M., Blank, L. M., and Rosenbaum, M. A. (2016). Strain- and substratedependent redox mediator and electricity production by Pseudomonas aeruginosa. Appl. Environ. Microbiol. 82, 5026-5038.doi: 10.1128/Aem.013 42-16

Bosire, E. M., and Rosenbaum, M. A. (2017). Electrochemical potential influences phenazine production, electron transfer and consequently electric current succinate in bioelectrochemical system of $E$. coli. This study highlights the ability to transfer electrons between electrode and microbial cells by engineering bacteria to produce self-excreted electronic mediators to facilitate bioproduction that is driven by bioelectrical currents. It provides ideas for the application of heterologous electron transfer mediator excreted by E. coli in MES or EF and lays a foundation for subsequent optimization.

\section{DATA AVAILABILITY STATEMENT}

The original contributions presented in the study are included in the article/Supplementary Material, further inquiries can be directed to the corresponding author.

\section{AUTHOR CONTRIBUTIONS}

JF and KC designed the project. JF, KL, and QL conducted and analyzed the experiments. SX provided technical assistance. JF analyzed the data and wrote the manuscript with input from SX, XW, KC, and PO. All authors reviewed and approved the manuscript.

\section{FUNDING}

This work was supported by the National Natural Science Foundation of China (Grant No. 21576134), National Science Foundation of Young Scientists of China (21908099), the National Key Research and Development Program of China (2016YFA0204300), Natural Science Research Projects of Colleges and Universities in Jiangsu Province (18KJB530009), the Jiangsu Synergetic Innovation Center for Advanced Bio-Manufacture (XTE1846), and Key Research and Development Program (Social Development) Project of Jiangsu Province (BE2018730).

\section{SUPPLEMENTARY MATERIAL}

The Supplementary Material for this article can be found online at: https://www.frontiersin.org/articles/10.3389/fbioe. 2020.590667/full\#supplementary-material

generation by Pseudomonas aeruginosa. Front. Microbiol. 8:892.doi: 10.3389/ Fmicb.2017.00892

Clark, I. C., Carlson, H. K., Iavarone, A. T., and Coates, J. D. (2012). Bioelectrical redox cycling of anthraquinone-2,6-disulfonate coupled to perchlorate reduction. Energy Environ. Sci. 5, 7970-7978.doi: 10.1039/c2ee21594b

Fang, X., Kalathil, S., Divitini, G., Wang, Q., and Reisner, E. (2020). A three-dimensional hybrid electrode with electroactive microbes for efficient electrogenesis and chemical synthesis. Proc. Natl. Acad. Sci. U.S.A. 117, 50745080.doi: 10.1073/pnas.1913463117

Feng, J., Jiang, M., Li, K., Lu, Q., Xu, S., Wang, X., et al. (2020). Direct electron uptake from a cathode using inward the Mtr pathway in Escherichia coli. Bioelectrochemistry 134:107498.doi: 10.1016/j.bioelechem.2020.107498

Feng, J., Qian, Y., Wang, Z., Wang, X., Xu, S., Chen, K. Q., et al. (2018). Enhancing the performance of Escherichia coli-inoculated microbial fuel cells 
by introduction of the phenazine-1-carboxylic acid pathway. J. Biotechnol. 275, 1-6.doi: 10.1016/j.jbiotec.2018.03.017

Freguia, S., Tsujimura, S., and Kano, K. (2010). Electron transfer pathways in microbial oxygen biocathodes. Electrochim. Acta 55, 813-818.doi: 10.1016/j. electacta.2009.09.027

Ganigue, R., Puig, S., Batlle-Vilanova, P., Balaguer, M. D., and Colprim, J. (2015). Microbial electrosynthesis of butyrate from carbon dioxide. Chem. Commun. 51, 3235-3238.doi: 10.1039/c4cc10121a

Harrington, T. D., Mohamed, A., Tran, V. N., Biria, S., Gargouri, M., Park, J. J., et al. (2015a). Neutral red-mediated microbial electrosynthesis by Escherichia coli, Klebsiella pneumoniae, and Zymomonas mobilis. Bioresour. Technol. 195, 57-65.doi: 10.1016/j.biortech.2015.06.005

Harrington, T. D., Tran, V. N., Mohamed, A., Renslow, R., Biria, S., Orfe, L., et al. (2015b). The mechanism of neutral red-mediated microbial electrosynthesis in Escherichia coli: menaquinone reduction. Bioresour. Technol. 192, 689-695.doi: 10.1016/j.biortech.2015.06.037

He, Z., and Mansfeld, F. (2009). Exploring the use of electrochemical impedance spectroscopy (EIS) in microbial fuel cell studies. Energy Environ. Sci. 2, 215219.doi: 10.1039/b814914c

Heering, H. A., Weiner, J. H., and Armstrong, F. A. (1997). Direct detection and measurement of electron relays in a multicentered enzyme: voltammetry of electrode-surface films of E-coli fumarate reductase, an iron-sulfur flavoprotein. J. Am. Chem. Soc. 119, 11628-11638.doi: 10.1021/Ja9723242

Heux, S., Cachon, R., and Dequin, S. (2006). Cofactor engineering in Saccharomyces cerevisiae: expression of a H2O-forming NADH oxidase and impact on redox metabolism. Metab. Eng. 8, 303-314.doi: 10.1016/j.ymben. 2005.12.003

Holloway, B. W., Krishnapillai, V., and Morgan, A. F. (1979). Chromosomal genetics of Pseudomonas. Microbiol Rev. 43, 73-102. doi: 10.1128/MMBR.43. 1.73- 102.1979

Jensen, H. M., Albers, A. E., Malley, K. R., Londer, Y. Y., Cohen, B. E., Helms, B. A., et al. (2010). Engineering of a synthetic electron conduit in living cells. Proc. Natl. Acad. Sci. U.S.A. 107, 19213-19218.doi: 10.1073/pnas.1009645107

Jiang, Y., Chen, B., Duan, C. L., Sun, B. B., Yang, J. J., and Yang, S. (2015). Multigene Editing in the Escherichia coli Genome via the CRISPR-Cas9 System. Appl. Environ. Microbiol. 81, 2506-2514.doi: 10.1128/Aem.04023-14

Kincaid, C. B., and Lavoie, K. H. (1989). Inhibition of bacterial growth in vitro following stimulation with high voltage, monophasic, pulsed current. Phys. Ther. 69, 651-655.doi: 10.1093/ptj/69.8.651

Liang, L. Y., Liu, R. M., Chen, X., Ren, X. Y., Ma, J. F., Chen, K. Q., et al. (2013). Effects of overexpression of NAPRTase, NAMNAT, and NAD synthetase in the $\mathrm{NAD}(\mathrm{H})$ biosynthetic pathways on the $\mathrm{NAD}(\mathrm{H})$ pool, $\mathrm{NADH} / \mathrm{NAD}(+)$ ratio, and succinic acid production with different carbon sources by metabolically engineered Escherichia coli. Biochem. Eng. J. 81, 90-96.doi: 10.1016/j.bej.2013. 09.018

Liu, H., Cheng, S. A., and Logan, B. E. (2005). Production of electricity from acetate or butyrate using a single-chamber microbial fuel cell. Environ. Sci. Technol. 39, 658-662.doi: 10.1021/es048927c

Liu, H., Matsuda, S., Hashimoto, K., and Nakanishi, S. (2012). Flavins secreted by bacterial cells of shewanella catalyze cathodic oxygen reduction. Chemsuschem 5, 1054-1058.doi: 10.1002/cssc.201100824
Nandy, A., Kumar, V., and Kundu, P. P. (2016). Effect of electric impulse for improved energy generation in mediatorless dual chamber microbial fuel cell through electroevolution of Escherichia coli. Biosens. Bioelectron. 79, 796801.doi: 10.1016/j.bios.2016.01.023

Nevin, K. P., Woodard, T. L., Franks, A. E., Summers, Z. M., and Lovley, D. R. (2010). Microbial electrosynthesis: feeding microbes electricity to convert carbon dioxide and water to multicarbon extracellular organic compounds. mBio 1:e00103-10.doi: 10.1128/mBio.00103-10

Patil, S. A., Hägerhäll, C., and Gorton, L. (2012). Electron transfer mechanisms between microorganisms and electrodes in bioelectrochemical systems. Bioanal. Rev. 4, 159-192.doi: 10.1007/s12566-012-0033-x

Rosenbaum, M., Aulenta, F., Villano, M., and Angenent, L. T. (2011). Cathodes as electron donors for microbial metabolism: which extracellular electron transfer mechanisms are involved? Bioresour. Technol. 102, 324-333.doi: 10.1016/j. biortech.2010.07.008

Schroder, U., Harnisch, F., and Angenent, L. T. (2015). Microbial electrochemistry and technology: terminology and classification. Energy Environ. Sci. 8, 513519.doi: 10.1039/c4ee03359k

Szuminsky, N. J., Albers, A. C., Unger, P., and Eddy, J. G. (1994). Effect of narrow, pulsed high voltages on bacterial viability. Phys. Ther. 74, 660-667.doi: 10.1093/ Ptj/74.7.660

Thrash, J. C., Van Trump, J. I., Weber, K. A., Miller, E., Achenbach, L. A., and Coates, J. D. (2007). Electrochemical stimulation of microbial perchlorate reduction. Environ. Sci. Technol. 41, 1740-1746.doi: 10.1021/ es062772m

Wang, H. M., and Ren, Z. Y. J. (2013). A comprehensive review of microbial electrochemical systems as a platform technology. Biotechnol. Adv. 31, 17961807.doi: 10.1016/j.biotechadv.2013.10.001

Wu, Z. Q., Wang, J. S., Liu, J., Wang, Y., Bi, C., and Zhang, X. L. (2019). Engineering an electroactive Escherichia coli for the microbial electrosynthesis of succinate from glucose and CO2. Microb. Cell Fact. 18:15.doi: 10.1186/s12934-0191067-3

Yang, Y., Ding, Y. Z., Hu, Y. D., Cao, B., Rice, S. A., Kjelleberg, S., et al. (2015). Enhancing bidirectional electron transfer of shewanella oneidensis by a synthetic flavin pathway. Acs Synth. Biol. 4, 815-823.doi: 10.1021/ sb500331x

Yong, Y. C., Yu, Y. Y., Li, C. M., Zhong, J. J., and Song, H. (2011). Bioelectricity enhancement via overexpression of quorum sensing system in Pseudomonas aeruginosa-inoculated microbial fuel cells. Biosen. Bioelectron. 30, 87-92.doi: 10.1016/j.bios.2011.08.032

Conflict of Interest: The authors declare that the research was conducted in the absence of any commercial or financial relationships that could be construed as a potential conflict of interest.

Copyright (C) 2020 Feng, $L u, L i, X u$, Wang, Chen and Ouyang. This is an open-access article distributed under the terms of the Creative Commons Attribution License (CC BY). The use, distribution or reproduction in other forums is permitted, provided the original author(s) and the copyright owner(s) are credited and that the original publication in this journal is cited, in accordance with accepted academic practice. No use, distribution or reproduction is permitted which does not comply with these terms. 